\title{
EXTREMAL BLACK HOLES AND ELEMENTARY STRING STATES
}

\author{
Ashoke Sen \\ International Centre for Theoretical Physics \\ P.O. Box 586, I-34100 Trieste, ITALY \\ and \\ Tata Institute of Fundamental Research \\ Homi Bhabha Road, Bombay 400005, INDIA \\ sen@theory.tifr.res.in, sen@tifrvax.bitnet
}

February 1, 2008

\begin{abstract}
Some of the extremal black hole solutions in string theory have the same quantum numbers as the Bogomol'nyi saturated elementary string states. We explore the possibility that these black holes can be identified to elementary string excitations. It is shown that stringy effects could correct the Bekenstein-Hawking formula for the black hole entropy in such a way that it correctly reproduces the logarithm of the density of elementary string states. In particular, this entropy has the correct dependence on three independent parameters, the mass and the left-handed charge of the black hole, and the string coupling constant.
\end{abstract}

hep-th/9504147

TIFR-TH-95-19

April, 1995 
There have been suggestions from diverse points of view that black holes should be treated as elementary particles[1, 2, 3, 4, 5, 6, 7, 8, 9, 10, 11]. The purpose of this paper is to investigate one aspect of this suggestion for a specific class of black holes in supersymmetric string theories 12], known as extremal black holes saturating the Bogomol'nyi bound 13.

Treating black holes as elementary particles poses a puzzle. In string theory there is an infinite tower of massive states which have the same quantum numbers as classical electrically charged black hole solutions. The question that arises naturally is, should we count the black holes and elementary string states as separate elementary particles? Or do they correspond to different ways of representing the same states? The second alternative certainly looks much more attractive. But to further substantiate this claim, one must show that the black holes have the same properties as elementary string states besides carrying the same quantum numbers.

One of the features which seems to be common between black holes and elementary string states is that for both the degeneracy of states with given mass (and charge) increases very rapidly with mass. For elementary string states this growth is due to the large number of oscillator states that correspond to a state with a given mass. For black holes, it arises due to the fact that the classical Bekenstein-Hawking entropy, which is proportional to the area of the event horizon, increases very rapidly with mass. Unfortunately, in trying to push this analogy further, we run into trouble. For elementary string states, the logarithm of the degeneracy of states increases linearly with mass, whereas the Bekenstein-Hawking entropy of the black hole increases as the square of the mass.

It has been suggested [4, 5] that this difficulty might be circumvented by taking into account the large renormalization of the mass of a black hole. In particular, it has been argued that black holes of mass $M^{2}$ should be identified to elementary string excitations of mass $M$. This would remove the discrepancy between the two entropies.

There are, however, some particular states in string theory, which do not receive any mass renormalization [14, 15]. These are the Bogomol'nyi saturated states alluded to earlier, and in comparing them to (extremal) black holes, we can no longer appeal to any possible renormalization of the mass. Thus these states provide a suitable laboratory for testing the hypothesis of black holes - elementary string excitations correspondence. It is for this reason that we shall focus our attention on these states in this paper. (This 
point has also been advocated by Vafa 16.)

How then are the entropies of extremal black holes related to the degeneracies of Bogomol'nyi saturated elementary string states? While the logarithm of the degeneracy of Bogomol'nyi saturated elementary string states continues to grow linearly with mass (although with a different proportionality factor), the area of the event horizon of an extremal black hole of the kind we are discussing actually vanishes. This is fortunate, since if it had not vanished, it would have almost certainly been proportional to the square of the black hole mass, leading to a contradiction, since now we can no longer appeal to a mass renormalization.t But now we have the opposite problem. The degeneracy associated with extremal black hole states seems to be smaller than the degeneracy of the elementary string states with the same quantum numbers.

To resolve this puzzle, we could postulate that the entropy of the extremal black hole is not exactly equal to the area of the event horizon, but the area of a surface close to the event horizon, which we shall call the 'stretched horizon'. This assumption is not totally unreasonable, since for various reasons we expect that our standard understanding of the physics of the black hole has to be modified very close to the horizon / singularity; and the stretched horizon represents a surface beyond which our standard understanding breaks down. The relevant question then is, what determines the location of the stretched horizon? Once we find an answer to this question, we can compute its area and study its relationship with the degeneracy of elementary string states. In particular, we can ask if it has the correct dependence on the mass and charge of the black hole, and the string coupling constant. The important point to note is that in order to carry out this comparison, we must define the location of the stretched horizon in a way that does not invole any unknown function of these three parameters. As we shall see, this can indeed be done.

We can explore different alternatives. If we believe that the necessary modification of the physics near the horizon comes from the string worldsheet effects, then we should define the stretched horizon to be the surface where the string world sheet theory becomes strongly coupled. In other

\footnotetext{
${ }^{1}$ It has however been argued $[17]$ that the original calculation of ref. $\left.\mid 18\right]$ has to be modified for calculating the entropy of an extremal black hole. The net result is that the entropy of an extremal black hole always vanishes, irrespective of whether the area of the event horizon vanishes or not. Since in the present case the area of the event horizon does vanish, we do not need to appeal to the arguments of ref. 17 .
} 
words, this is the surface where the space-time curvature associated with the string metric, and/or other target space field strengths, becomes large. We shall see that this definition determines the location of the stretched horizon uniquely up to a purely numerical factor.

We might also try to define the stretched horizon to be the place where the string coupling constant becomes large. However, for black holes of the type we shall discuss, this does not happen. In fact, the string coupling vanishes as we approach the event horizon. Thus if we start from a configuration where the asymptotic value of the string coupling is small, then it remains small everywhere in space-time, and as a result we cannot define the stretched horizon using the criteria of string coupling becoming strong.

The third possibility makes use of black hole thermodynamics, and is in fact the original definition of stretched horizon given in ref.[4, 19]. According to this definition, the stretched horizon is defined to be the surface where the local Unruh temperature for an observer, who is stationary in the Schwarzschild coordinate, is of the order of the Hagedorn temperature of the string theory. $^{2}$ This surface is close to the event horizon, where the local Unruh temperature is infinite. As we shall see, this definition actually coincides with the first definition of the stretched horizon for electrically charged extremal black holes.

In the rest of this paper, we shall carry out a careful calculation of the area of the stretched horizon, defined as above, as a function of the mass and charge of the black hole, as well as the string coupling constant, and show that the result agrees with the logarithm of the degeneracy of the elementary string states. [ We shall also discuss how stringy effects might produce such a modification of the Bekenstein-Hawking formula. Throughout this paper we shall work with the four dimensional theory obtained by toroidal compactification of the heterotic string theory [21], and use the normalization conventions of ref.[22].

We begin by writing down the effective action describing the dynamics of the massless fields in four dimensions:

$$
S=\frac{1}{32 \pi} \int d^{4} x \sqrt{-G} e^{-\Phi}\left[R_{G}+G^{\mu \nu} \partial_{\mu} \Phi \partial_{\nu} \Phi-\frac{1}{12} G^{\mu \mu^{\prime}} G^{\nu \nu^{\prime}} G^{\rho \rho^{\prime}} H_{\mu \nu \rho} H_{\mu^{\prime} \nu^{\prime} \rho^{\prime}}\right.
$$

\footnotetext{
${ }^{2}$ It has now been demonstrated[20] that the conventional treatment of black hole thermodynamics does break down beyond this surface.

${ }^{3} \mathrm{~A}$ preliminary version of this result was stated briefly in ref.[7].
} 


$$
\left.-G^{\mu \mu^{\prime}} G^{\nu \nu^{\prime}} F_{\mu \nu}^{(a)}(L M L)_{a b} F_{\mu^{\prime} \nu^{\prime}}^{(b)}+\frac{1}{8} G^{\mu \nu} \operatorname{Tr}\left(\partial_{\mu} M L \partial_{\nu} M L\right)\right] .
$$

Here $G_{\mu \nu}, B_{\mu \nu}$ and $A_{\mu}^{(a)}(0 \leq \mu, \nu \leq 3,1 \leq a \leq 28)$ are the string metric, anti-symmetric tensor fields, and $U(1)^{28}$ gauge fields respectively, $\Phi$ is the dilaton, $R_{G}$ is the scalar curvature associated with the metric $G_{\mu \nu}$, and,

$$
\begin{aligned}
F_{\mu \nu}^{(a)} & =\partial_{\mu} A_{\nu}^{(a)}-\partial_{\nu} A_{\mu}^{(a)}, \\
H_{\mu \nu \rho} & =\left(\partial_{\mu} B_{\nu \rho}+2 A_{\mu}^{(a)} L_{a b} F_{\nu \rho}^{(b)}\right)+\text { cyclic permutations of } \mu, \nu, \rho
\end{aligned}
$$

are the field strengths associated with $A_{\mu}^{(a)}$ and $B_{\mu \nu} . M$ is a $28 \times 28$ matrix valued scalar field, satisfying,

$$
M L M^{T}=L, \quad M^{T}=M,
$$

and,

$$
L=\left(\begin{array}{cc}
-I_{22} & 0 \\
0 & I_{6}
\end{array}\right),
$$

where $I_{n}$ denotes $n \times n$ identity matrix. The action (四) is invariant under an $\mathrm{O}(6,22)$ transformation:

$$
\begin{aligned}
& M \rightarrow \Omega M \Omega^{T}, \quad A_{\mu}^{(a)} \rightarrow \Omega_{a b} A_{\mu}^{(b)}, \\
& G_{\mu \nu} \rightarrow G_{\mu \nu}, \quad \Phi \rightarrow \Phi, \quad B_{\mu \nu} \rightarrow B_{\mu \nu},
\end{aligned}
$$

where $\Omega$ is a $28 \times 28$ matrix satisfying,

$$
\Omega L \Omega^{T}=L .
$$

This remains a valid symmetry of the action even after we include the higher derivative terms in the action originating from the higher order corrections in the string world-sheet theory. This fact will be useful to us later.

We also define the canonical Einstein matric $g_{\mu \nu}$ as follows:

$$
g_{\mu \nu}=e^{-\Phi} G_{\mu \nu} .
$$

In terms of $g_{\mu \nu}$, the action takes the form:

$$
\begin{aligned}
S= & \frac{1}{32 \pi} \int d^{4} x \sqrt{-g}\left[R_{g}-\frac{1}{2} g^{\mu \nu} \partial_{\mu} \Phi \partial_{\nu} \Phi-\frac{1}{12} e^{-2 \Phi} g^{\mu \mu^{\prime}} g^{\nu \nu^{\prime}} g^{\rho \rho^{\prime}} H_{\mu \nu \rho} H_{\mu^{\prime} \nu^{\prime} \rho^{\prime}}\right. \\
& \left.-e^{-\Phi} g^{\mu \mu^{\prime}} g^{\nu \nu^{\prime}} F_{\mu \nu}^{(a)}(L M L)_{a b} F_{\mu^{\prime} \nu^{\prime}}^{(b)}+\frac{1}{8} g^{\mu \nu} \operatorname{Tr}\left(\partial_{\mu} M L \partial_{\nu} M L\right)\right] .
\end{aligned}
$$


As in ref.[22], we use the normalization $\alpha^{\prime}=16$. This corresponds to a string world-sheet action of the form:

$$
\frac{1}{64 \pi} \int d^{2} \xi G_{\mu \nu}(X) \partial_{\alpha} X^{\mu} \partial^{\alpha} X^{\nu}+\cdots
$$

where $\cdots$ denotes terms involving fermionic fields on the world sheet, as well as the target space fields $B_{\mu \nu}, A_{\mu}^{(a)}, \Phi$ and $M$. We shall restrict ourselves to backgrounds characterized by the follwing asymptotic forms of various fields:

$\left\langle g_{\mu \nu}\right\rangle=\eta_{\mu \nu}, \quad\left\langle e^{-\Phi}\right\rangle=\frac{1}{g^{2}}, \quad\langle M\rangle=I_{28}, \quad\left\langle B_{\mu \nu}\right\rangle=0, \quad\left\langle A_{\mu}^{(a)}\right\rangle=0$.

This gives

$$
\left\langle G_{\mu \nu}\right\rangle=g^{2} \eta_{\mu \nu} .
$$

From eqs.(8), (9) and (11) we see that the background we are using corresponds to the following values of the Newton's constant $G_{N}$ and string tension $T$ measured by an asymptotic observer:

$$
G_{N}=2, \quad T=\frac{g^{2}}{32 \pi} .
$$

Most general electrically charged rotating black hole solutions in this theory were constructed in ref. [7]. (See also [23, 24].) We shall specialize on the non-rotating extremal black holes saturating the Bogomol'nyi bound. The most general black hole solution of this type is given by.

$$
\begin{gathered}
d s^{2} \equiv g_{\mu \nu} d x^{\mu} d x^{\nu} \\
=-K^{-1 / 2} \rho d t^{2}+K^{1 / 2} \rho^{-1} d \rho^{2}+K^{1 / 2} \rho\left(d \theta^{2}+\sin ^{2} \theta d \phi^{2}\right), \\
B_{\mu \nu}=0, \\
e^{\Phi}=K^{-1 / 2} \rho g^{2}, \\
A_{t}^{(a)=}-g \frac{n^{(a)}}{\sqrt{2}} \frac{m_{0}}{K} \rho \sinh \alpha, \quad \text { for } \quad 1 \leq a \leq 22, \\
=-g \frac{p^{(a-22)}}{\sqrt{2}} \frac{m_{0}}{K}\left(\rho \cosh \alpha+m_{0}\right), \quad \text { for } \quad 23 \leq a \leq 28,
\end{gathered}
$$

\footnotetext{
${ }^{4}$ Ref. [7] constructed the solution for $g=1$. The solution for a general $g$ can easily be obtained from there by appropriate rescaling of $e^{-\Phi}$ and $A_{\mu}^{(a)}$.
} 


$$
M=I_{28}+\left(\begin{array}{cc}
P n n^{T} & Q n p^{T} \\
Q p n^{T} & P p p^{T}
\end{array}\right)
$$

where $m_{0}$ and $\alpha$ are two real numbers, $\vec{n}$ is a 22 dimensional unit vector, $\vec{p}$ is a 6 dimensional unit vector, and,

$$
\begin{gathered}
K=\left(\rho^{2}+2 m_{0} \rho \cosh \alpha+m_{0}^{2}\right), \\
P=2 \frac{m_{0}^{2}}{K} \sinh ^{2} \alpha, \\
Q=-2 \frac{m_{0} \sinh \alpha}{K}\left(\rho+m_{0} \cosh \alpha\right) .
\end{gathered}
$$

The horizon and the singularity of this black hole coincide, both being situated at $\rho=0$.

We define the electric charge $Q_{e l}^{(a)}$ carried by the black hole through the equations:

$$
F_{\rho t}^{(a)} \simeq \frac{Q_{e l}^{(a)}}{\rho^{2}} \quad \text { for large } \rho
$$

Eq.(16) then gives

$$
\begin{aligned}
Q_{e l}^{(a)} & =g \frac{n^{(a)}}{\sqrt{2}} m_{0} \sinh \alpha, \quad \text { for } \quad 1 \leq a \leq 22, \\
& =g \frac{p^{(a-22)}}{\sqrt{2}} m_{0} \cosh \alpha, \quad \text { for } \quad 23 \leq a \leq 28 .
\end{aligned}
$$

Also, from eqs.(13), (18) we see that the ADM mass of the black hole is given by,

$$
m=\frac{1}{G_{N}} \frac{m_{0}}{2} \cosh \alpha=\frac{1}{4} m_{0} \cosh \alpha .
$$

It is customary to define the left and right components of the electric charge vector as follows:

$$
\begin{aligned}
Q_{R}^{(a)} & =0 \text { for } \quad 1 \leq a \leq 22, \\
& =g \frac{p^{(a-22)}}{\sqrt{2}} m_{0} \cosh \alpha \quad \text { for } \quad 23 \leq a \leq 28, \\
Q_{L}^{(a)} & =g \frac{n^{(a)}}{\sqrt{2}} m_{0} \sinh \alpha \quad \text { for } \quad 1 \leq a \leq 22, \\
& =0 \text { for } \quad 23 \leq a \leq 28 .
\end{aligned}
$$


From eqs.(22) and (23) we get,

$$
m^{2}=\frac{1}{8 g^{2}} \vec{Q}_{R}^{2}
$$

which is the standard Bogomol'nyi relation between mass and charge. The independent parameters characterizing the black hole may be taken to be $m$, $Q_{L} \equiv\left|\vec{Q}_{L}\right|, \vec{n}$ and $\vec{p}$. Eqs.(22), (23) may now be inverted to give,

$$
m_{0}=4 \sqrt{m^{2}-\frac{\vec{Q}_{L}^{2}}{8 g^{2}}}, \quad \alpha=\tanh ^{-1}\left(\frac{Q_{L}}{2 \sqrt{2} g m}\right) .
$$

We shall now determine the position of the stretched horizon by examining various fields close to the horizon. For this we note that near the horizon $\left(\rho<<m_{0}\right)$, the string metric takes the form:

$$
\begin{aligned}
d S^{2} & \equiv G_{\mu \nu} d x^{\mu} d x^{\nu}=e^{\Phi} d s^{2} \\
& \simeq-\frac{\rho^{2}}{m_{0}^{2}} g^{2} d t^{2}+g^{2} d \rho^{2}+g^{2} \rho^{2}\left(d \theta^{2}+\sin ^{2} \theta d \phi^{2}\right) .
\end{aligned}
$$

In the new coordinate system

$$
\bar{\rho}=g \rho, \quad \bar{t}=t / m_{0},
$$

the metric takes the form:

$$
d S^{2} \simeq-\bar{\rho}^{2} d \bar{t}^{2}+d \bar{\rho}^{2}+\bar{\rho}^{2}\left(d \theta^{2}+\sin ^{2} \theta d \phi^{2}\right) .
$$

In the same coordinate system, other non-trivial fields near the horizon are

$$
\begin{aligned}
& \partial_{\bar{\rho}} \Phi \simeq \frac{1}{\bar{\rho}}, \\
& F_{\bar{\rho} \bar{t}}^{(a)} \simeq-\frac{n^{(a)}}{\sqrt{2}} \sinh \alpha \quad \text { for } \quad 1 \leq a \leq 22, \\
& \simeq \frac{p^{(a-22)}}{\sqrt{2}} \cosh \alpha \quad \text { for } \quad 23 \leq a \leq 28 \text {. } \\
& M=I_{28}+\left(\begin{array}{cc}
2 \sinh ^{2} \alpha n n^{T} & -2 \sinh \alpha \cosh \alpha n p^{T} \\
-2 \sinh \alpha \cosh \alpha p n^{T} & 2 \sinh ^{2} \alpha p p^{T}
\end{array}\right) .
\end{aligned}
$$


Note that in this coordinate system, all dependence of the background fields on the parameters $m_{0}$ and $g$ has disappeared, but $F_{\bar{\rho} \bar{t}}^{(a)}$ and $M$ still depend on $\alpha$. However, the $\alpha$ dependence of this background can be removed by making an $O(6,22)$ transformation (5) with the matrix

$$
\Omega=\left(\begin{array}{cc}
\cosh \alpha n n^{T} & \sinh \alpha n p^{T} \\
\sinh \alpha p n^{T} & \cosh \alpha p p^{T}
\end{array}\right) .
$$

This gives,

$$
\begin{aligned}
M^{\prime} & \simeq I_{28} \\
F_{\bar{\rho} \bar{t}}^{\prime(a)} & \simeq 0 \quad \text { for } \quad 1 \leq a \leq 22, \\
& \simeq \frac{p^{(a-22)}}{\sqrt{2}} \quad \text { for } \quad 23 \leq a \leq 28 .
\end{aligned}
$$

Since (5) represents a symmetry of the full effective action at the string tree level, effects of world sheet quantum corrections, which show up as higher derivative terms in the effective action, could be studied as well in the transformed background represented by the primed fields. Since the background fields now do not depend on any parameter, we see that the place where the target space field strengths become strong is situated unambiguously at $\bar{\rho} \sim 1$. This determines the location of the stretched horizon to be at

$$
\bar{\rho}=C,
$$

where $C$ is a pure number. This gives,

$$
\rho=C / g \equiv \eta .
$$

It has been shown in the appendix that at this value of $\rho$, the local Unruh temperature is of the order of the Hagedorn temperature in string theory. Thus the two definitions of stretched horizon coincide.

The area of the stretched horizon, calculated using the canonical metric (13) is given by,

$$
A \simeq 4 \pi \eta m_{0}=4 \pi m_{0} C / g .
$$

Following the Bekenstein-Hawking result, we define the entropy of the black hole to be

$$
S_{B . H .} \equiv \frac{A}{4 G_{N}}=\frac{\pi}{2} \frac{m_{0} C}{g}=\frac{2 \pi C}{g} \sqrt{m^{2}-\frac{\vec{Q}_{L}^{2}}{8 g^{2}}},
$$


where we have used eq.(25).

Let us examine this equation in some detail. What kind of world sheet corrections could produce such a modification of the entropy formula? For this, we look at the euclidean version of the string metric and the dilaton near the horizon:

$$
\begin{aligned}
d S_{E}^{2} & \simeq \bar{\rho}^{2} d \bar{\tau}^{2}+d \bar{\rho}^{2}+\bar{\rho}^{2}\left(d \theta^{2}+\sin ^{2} \theta d \phi^{2}\right) \\
e^{\Phi} & \simeq m_{0}^{-1} g \bar{\rho}
\end{aligned}
$$

where $\bar{\tau}=-i \bar{t}$. Let us define

$$
\bar{\Phi}=\Phi-\ln \left(g / m_{0}\right), \quad \bar{g}_{\mu \nu}=e^{-\bar{\Phi}} G_{\mu \nu},
$$

and introduce a new coordinate $\bar{r}$ through the relation

$$
\bar{\rho}=\bar{r}^{2} / 4 \text {. }
$$

Then eq.(38) can be rewritten as

$$
\begin{aligned}
d \bar{s}_{E}^{2} & \equiv \bar{g}_{\mu \nu} d x^{\mu} d x^{\nu} \\
& \simeq \frac{1}{4} \bar{r}^{2} d \bar{\tau}^{2}+d \bar{r}^{2}+\frac{1}{4} \bar{r}^{2}\left(d \theta^{2}+\sin ^{2} \theta d \phi^{2}\right) \\
e^{\bar{\Phi}} & \simeq \bar{r}^{2} / 4
\end{aligned}
$$

If we take the coordinate $\bar{\tau}$ to be periodic with period $4 \pi$, then the $\bar{r}, \bar{\tau}$ part of the metric is non-singular at $\bar{r}=0$; however the full metric is singular since the area of the transverse sphere spanned by $\theta, \phi$ vanishes as $\bar{r} \rightarrow 0$. Furthermore, the dilaton also becomes singular at $\bar{r}=0$. Let us suppose that the strong coupling effects on the world sheet render the solution finite at $\bar{r}=0$ by modifying the metric in such a way that the area of the transverse sphere does not collapse at $\bar{r}=0$, and the dilaton does not blow up as $\bar{r} \rightarrow 0$. $]$ This modification must be such that it vanishes for $\bar{r}>>1$. A

\footnotetext{
${ }^{5}$ It has been argued in ref. [25] that perturbative corrections on the world-sheet does not modify the lowest order solution. However this statement is valid only for a particular renormalization scheme in the world sheet theory, and the metric that is relevant for the entropy calculation may not be the one used in ref.25. Put another way, if we insist on working with the metric of ref. 25] then the surface term relevant for entropy calculation may be modified by higher derivative terms in the effective action.
} 
possible modification of the solution near the point $\bar{r}=0$, that does this, is of the form:

$$
\begin{aligned}
d \bar{s}_{E}^{2} & \simeq \frac{1}{4} \bar{r}^{2} d \bar{\tau}^{2}+d \bar{r}^{2}+f_{1}(\bar{r})\left(d \theta^{2}+\sin ^{2} \theta d \phi^{2}\right) \\
e^{\bar{\Phi}} & \simeq f_{2}(\bar{r})
\end{aligned}
$$

where $f_{1}$ and $f_{2}$ are two functions of $\bar{r}$ such that $f_{1}(\bar{r}) \simeq \bar{r}^{2} / 4, f_{2}(\bar{r}) \simeq \bar{r}^{2} / 4$ for $\bar{r}>>1$, and $f_{1}(0), f_{2}(0)$ are finite and non-zero. The important point to note is that $f_{1}$ and $f_{2}$ do not depend on any of the parameters $m_{0}, \alpha$ or $g$. This solution is non-singular if $\bar{\tau}$ is taken to be a periodic coordinate with period $4 \pi$. The canonical metric $g_{\mu \nu}=\left(m_{0} / g\right) \bar{g}_{\mu \nu}$ now takes the form:

$$
d s_{E}^{2} \simeq \frac{m_{0}}{g}\left(\frac{1}{4} \bar{r}^{2} d \bar{\tau}^{2}+d \bar{r}^{2}+f_{1}(\bar{r})\left(d \theta^{2}+\sin ^{2} \theta d \phi^{2}\right)\right) .
$$

Near $\bar{r}=0$, the geometry of the space is that of a disc times a sphere of radius

$$
\sqrt{\frac{m_{0} f_{1}(0)}{g}} .
$$

Then the entropy of the black hole, which is $\left(4 G_{N}\right)^{-1}$ times the area of this sphere, is given by,

$$
\frac{\pi m_{0} f_{1}(0)}{2 g}=\frac{2 \pi f_{1}(0)}{g} \sqrt{m^{2}-\frac{\vec{Q}_{L}^{2}}{8 g^{2}}} .
$$

This has the same form as the expression for $S_{B . H}$. given in eq.(37).

Although for this calculation we have postulated a specific scenario, it should be clear from this discussion that any modification of the surface terms contributing to the entropy due to the turning on of the stringy effects at $\bar{\rho} \sim 1$, will give an expression for the entropy of the form of (37). The main point is that the $\exp (-\Phi)$ term in front of the action will produce a factor of $\left(m_{0} / g\right)$, and the rest of the surface terms, which depend on the combination $\left(g / m_{0}\right) \exp (-\Phi)$, the string metric and the other field strengths at $\bar{\rho} \sim 1$, will be totally independent of the parameters $m_{0}, \alpha$ and $g$. Thus the

\footnotetext{
${ }^{6}$ Besides the extra surface terms that need to be added to the action 18$]$, the contribution from the bulk action is also given by a surface term on shell, since $S=$ $-\int d^{4} x(\delta S / \delta \Phi(x))=-\int d^{4} x \partial_{\mu}\left(\delta S / \delta\left(\partial_{\mu} \Phi(x)\right)\right)$.
} 
contribution to the entropy will be proportional to $m_{0} / g$, in agreement with eq.(37). This shows that if there is a modification of the Bekenstein-Hawking formula for the black hole entropy due to string world-sheet effects, then the correction is naturally of the form given in eq.(37).

We shall now compare the expression for the entropy given in eq.(37) with the density of elementary string states with the same mass and charge quantum numbers. In the normalization convention we are using, the mass formula for the Bogomol'nyi saturated elementary string states is given by,

$$
m^{2}=\frac{\vec{Q}_{R}^{2}}{8 g^{2}}=\frac{g^{2}}{8}\left(\frac{\vec{Q}_{L}^{2}}{g^{4}}+2 N_{L}-2\right),
$$

where $N_{L}$ is the total oscillator contribution to the squared mass from the left moving oscillators. There is no contribution to the mass from the right moving oscillators, since in order to saturate the Bogomol'nyi bound, the string state must be at the lowest level in the right moving sector of the worldsheet. The degeneracy of such states arises due to the many different ways the left moving oscillators make up the total number $N_{L}$. This degeneracy has been calculated many times (for a recent calculation, see [5]) and is given by,

$$
d_{\text {E.S. }} \simeq \exp \left(4 \pi \sqrt{N_{L}}\right) .
$$

Thus, the entropy, calculated from the elementary string spectrum, is given by,

$$
S_{E . S .} \equiv \ln d_{E . S .} \simeq 4 \pi \sqrt{N_{L}} \simeq \frac{8 \pi}{g} \sqrt{m^{2}-\frac{\vec{Q}_{L}^{2}}{8 g^{2}}} .
$$

This has the same dependence on $g, m$ and $\vec{Q}_{L}$ as $S_{B . H}$. given in eq.(37). The overall constant of proportionality in the two expressions agree if we make the choice

$$
C=4 \text {. }
$$

This shows that the modified Bekenstein-Hawking entropy of the black hole does reproduce the density of elementary string states correctly.

Acknowledgement: I wish to thank John Schwarz for several illuminating discussions, without which the detailed computation given here would not have been performed. Some of these results were reported at the workshop on 'Physics at the Planck Scale, Puri, India, Dec.12-21, 1994'. I would like to thank the organisers of the workshop for hospitality. 


\section{A Stretched Horizon and Black Hole Ther- modynamics}

In this appendix we shall show that at the location of the stretched horizon defined in eq.(35), the local Unruh temperature does become of the order of the Hagedorn temperature of string theory. This would show that our definition of the stretched horizon agrees with the definition of the stretched horizon advocated in ref. [4]. To do this we first euclideanize the canonical metric (13), and also choose an appropriate coordinate system in which the $\rho, t$ part of the metric is non-singular. This is done through the replacement

$$
t=i \tau, \quad \rho=r^{2} .
$$

The canonical metric near $\rho=0$ then takes the form

$$
d s_{E}^{2} \simeq 4 m_{0}\left(d r^{2}+\frac{r^{2}}{4 m_{0}^{2}} d \tau^{2}\right)+m_{0} r^{2}\left(d \theta^{2}+\sin ^{2} \theta d \phi^{2}\right)
$$

The $r, \tau$ part of the metric describes a non-singular space, provided $\tau$ describes a periodic coordinate with period $4 \pi m_{0}$ :

$$
\tau \equiv \tau+4 \pi m_{0}
$$

From now on we shall make this identification.

The inverse of the the local Unruh temperature is the proper period in the $\tau$ direction at a fixed value of $\rho$ (or $r$ ). Using the metric (A.2), we see that at $\rho=\eta$ this is given by

$$
\beta_{U n r u h}(\eta) \simeq 4 \pi \sqrt{m_{0} \eta}=4 \pi \sqrt{\frac{m_{0} C}{g}},
$$

where we have used the value of $\eta$ given in eq.(35).

In order to calculate the local Hagedorn temperature, we need to take into

account the fact that the string coupling, labelled by $e^{\Phi}$, is not a constant in the black hole background, but actually varies with $\rho$. In fact, at $\rho=\eta$ it is given by,

$$
e^{\Phi(\eta)} \simeq g^{2} \eta / m_{0}=C g / m_{0}
$$


This gives

$$
\beta_{\text {Hagedorn }}(\eta)=4 \pi(2+\sqrt{2}) e^{-\Phi(\eta) / 2}=4 \pi(2+\sqrt{2}) \sqrt{\frac{m_{0}}{C g}} .
$$

Since $C \sim 1$ we see that at the stretched horizon, the local Unruh temperature is indeed of the same order as the local Hagedorn temperature.?

\section{References}

[1] S. Hawking, Monthly Notices Roy. Astron. Soc. 152 (1971) 75;

A. Salam, in Quantum Gravity: an Oxford Symposium (eds. Isham, Penrose and Sciama, O.U.P. 1975).

[2] G. 't Hooft, Nucl. Phys. B335 (1990) 138.

[3] C. Holzhey and F. Wilczek, Nucl. Phys. B380 (1992) 447 hepth/9202014].

[4] L. Susskind, 'Some speculations about black hole entropy in string theory', preprint RU-93-44 hep-th/9309145;

L. Susskind and J. Uglum, Phys. Rev. D50 (1994) 2700 thepth/9401070].

[5] J. Russo and L. Susskind, 'Asymptotic level density in heterotic string theory and rotating black holes', preprint UTTG-9-94 hep-th/9405117.

[6] M. Duff, R. Khuri, R. Minasian and J. Rahmfeld, Nucl. Phys. B418 (1994) 195 [hep-th/9311120;

M. Duff and J. Rahmfeld, Phys. Lett. B345 (1995) 441 hepth/9406105].

[7] A. Sen, 'Black hole solutions in heterotic string theory on a torus', preprint TIFR-TH-94-47 [hep-th/9411187.

\footnotetext{
${ }^{7}$ Note that since the string coupling vanishes as we approach the horizon, the modification of the phase transition temperature due to the effects discussed in ref. [26] is expected to be small.
} 
[8] C. Hull and P. Townsend, Nucl. Phys. B438 (1995) 109 hepth/9410167];

P. Townsend, 'The eleven dimensional super-membrane revisited', preprint DAMTP/R/95/2 hep-th/9501068.

[9] E. Witten, 'String theory dynamics in various dimensions', preprint IASSNS-HEP-95-18 hep-th/9503124.

[10] A. Strominger, 'Massless black holes and conifolds in string theory', preprint hep-th/9504090

B. Greene, D. Morrison and A. Strominger, 'Black hole condensation and the unification of string vacua', preprint CLNS-95/1335 hepth/9504145].

[11] A. Burinskii, 'Some properties of the Kerr solution to the low energy string theory', preprint IBRAE-95-08 hep-th/9504139;

H. Nishino, 'Stationary axi-symmetric black holes, $\mathrm{N}=2$ superstring, and self-dual gauge or gravity fields', preprint UMDEPP 95-111 hepth/9504142].

[12] G. Horowitz, 'The dark side of string theory: Black holes and black strings', in 'Trieste 1992, Proceedings, String Theory and Quantum Gravity '92', p55 hep-th/9210119;

A. Sen, 'Black holes and solitons in string theory', in 'Pathways to Fundamental Theories, Proceedings, Johns Hopkins Workshop, Goteborg, '92', p 155 hep-th/9210050;

B. Campbell, N. Kaloper, R. Madden and K. Olive, Nucl. Phys. B399 (1993) 137 hep-th/9301129;

and references therein.

[13] G. Gibbons and C. Hull, Phys. Lett. B109 (1982) 109;

J. Harvey and J. Liu, Phys. Lett. B268 (1991) 40;

R. Kallosh, A. Linde, T. Ortin, A. Peet and A. Van Proeyen, Phys. Rev. D46 (1992) 5278 hep-th/9205027;

[14] E. Witten and D. Olive, Phys. Lett. B78 (1978) 97.

[15] R. Kallosh, Phys. Lett. B282 (1992) 80. 
[16] C. Vafa, as quoted in first reference in [4].

[17] S. Hawking, G. Horowitz and S. Ross, 'Entropy, area and black hole pairs', preprint DAMTP/R 94-26 gr-qc/9409013;

G. Gibbons and R. Kallosh, Phys. Rev. D51 (1995) 2839 hepth/9407118;

C. Teitelboim, 'Action and entropy of extreme and non-extreme black holes', preprint IASSNS-HEP-94-84 hep-th/9410103;

S. Hawking and G. Horowitz, 'The gravitational hamiltonian, action, entropy, and surface terms', preprint DAMTP-R-94-52 gr-qc/9501014.

[18] G. Gibbons and S. Hawking, Phys. Rev. D15 (1977) 2752, Comm. Math. Phys. 66 (1979) 291.

[19] L. Susskind, Phys. Rev. D49 (1994) 6606 hep-th/9308139.

[20] J. Barbon, Phys. Lett. B339 (1994) 41 hep-th/9406209;

A. Dabholkar, Phys. Lett. B347 (1995) 222 hep-th/9409158.

[21] K. Narain, Phys. Lett. B169 (1986) 41;

K. Narain, H. Sarmadi and E. Witten, Nucl. Phys. B279 (1987) 369.

[22] A. Sen, Int. J. Mod. Phys. A9 (1994) 3707 hep-th/9402002 .

[23] P. Breitenlohner, G. Gibbons and D. Maison, Comm. Math. Phys. 120 (1988) 295.

[24] M. Cvetic and D. Youm, Nucl. Phys. B438 (1995) 182; preprints hepth/9502119, hep-th/9503081.

[25] G. Horowitz and A. Tseytlin, Phys. Rev. Lett. 73 (1994) 3351 hepth/9408040].

[26] J. Atick and E. Witten, Nucl. Phys. B310 (1988) 291. 